\title{
Regorafenib induced severe toxic hepatitis: characterization and discussion
}

Sacré, Anne ; Lanthier, Nicolas ; Dano, Hélène ; Aydin, Selda ; Leggenhager, Daniela ; Weber, Achim ; Dekairelle, Anne-France ; De Cuyper, Astrid ; Gala, Jean-Luc ; Humblet, Yves ; Sempoux, Christine ; Van den Eynde, Marc

\begin{abstract}
BACKGROUND Regorafenib is the first small-molecule multikinase inhibitor which showed survival benefits in pretreated metastatic colorectal cancer (mCRC) patients. Beside classical adverse events of this drug class, hepatotoxicity has been described as a frequent side effect. MATERIAL AND METHODS Patients with refractory $\mathrm{mCRC}$ treated with regorafenib in our institution were reviewed. Severe treatment-related liver toxicity was investigated. Clinical history, liver histology and genetic assessment (sequence analysis) of cytochrome P 3A4 (CYP3A4) and uridine diphosphate-glucuronosyltransferase 1A9 (UGT1A9) involved in regorafenib metabolization were here reported for patients with severe hepatotoxicity. RESULTS Among the 93 reviewed patients, 3 presented severe and icteric toxic hepatitis which was fatal for 1 patient. Histopathological liver lesions were different depending on the onset of hepatotoxicity (acute or subacute): acinar zone 3 necrosis in case of acute symptoms and portal tract inflammation with porto-central bridging and fibrosis in the delayed presentation. None patients had CYP3A4 gene mutations. Similar polymorphisms in UGT1A9 gene promoter region (UGT1A9 variant -118T9>10 (rs3832043)) were found in both patients who presented acute hepatitis. Moreover, it appears retrospectively that both of them already experienced significant toxicity under irinotecan-based chemotherapy. CONCLUSION This is the first report of severe hepatotoxicity with available liver histology and genetic assessment of enzymes involved in regorafenib metabolization. This report also reminds the importance of a close liver tests monitoring during regorafenib treatment. This article is protected by copyright. All rights reserved.
\end{abstract}

DOI: https://doi.org/10.1111/liv.13217

Posted at the Zurich Open Repository and Archive, University of Zurich

ZORA URL: https://doi.org/10.5167/uzh-125658

Journal Article

Accepted Version

Originally published at:

Sacré, Anne; Lanthier, Nicolas; Dano, Hélène; Aydin, Selda; Leggenhager, Daniela; Weber, Achim; Dekairelle, Anne-France; De Cuyper, Astrid; Gala, Jean-Luc; Humblet, Yves; Sempoux, Christine; Van den Eynde, Marc (2016). Regorafenib induced severe toxic hepatitis: characterization and discussion. Liver International:15.

DOI: https://doi.org/10.1111/liv.13217 
Received Date : 16-Mar-2016

Revised Date : 22-Jul-2016

Accepted Date : 28-Jul-2016

Article type : Rapid Communication

Editor : Raúl Andrade

\section{Regorafenib induced severe toxic hepatitis: characterization and discussion.}

Anne Sacré ${ }^{1}$, Nicolas Lanthier ${ }^{2}$, Hélène Dano ${ }^{3}$, Selda Aydin ${ }^{3}$, Daniela Leggenhager ${ }^{4}$, Achim Weber ${ }^{4}$, Anne-France Dekairelle ${ }^{5}$, Astrid De Cuyper ${ }^{1}$, Jean-Luc Gala ${ }^{5}$, Yves Humblet ${ }^{1}$, Christine Sempoux ${ }^{6}$, Marc Van den Eynde ${ }^{1,2}$.

${ }^{1}$ Service d'Oncologie médicale, Cliniques Universitaires Saint-Luc, Université catholique de Louvain (UCL), Brussels, Belgium.

${ }^{2}$ Service d'Hépato-gastroentérologie, Cliniques Universitaires Saint-Luc, UCL, Brussels, Belgium

${ }^{3}$ Service d'anatomie pathologique, Cliniques Universitaires Saint-Luc, UCL, Brussels, Belgium

${ }^{4}$ Institute of Surgical Pathology, University Zurich, Switzerland

${ }^{5}$ Centre de Technologies Moléculaires Appliquées, Cliniques Universitaires Saint-Luc, UCL, Brussels, Belgium.

${ }^{6}$ Institut Universitaire de Pathologie-IUP, Lausanne, Switzerland

\section{Corresponding Author:}

Marc Van den Eynde

Avenue Hippocrate 10

B- 1200 Bruxelles

Telephone: 003227641041

marc.vandeneynde@uclouvain.be

\section{Main body of manuscript :1973 words}

1 figure, 3 tables ( 2 supplementary).

This article has been accepted for publication and undergone full peer review but has not been through the copyediting, typesetting, pagination and proofreading process, which may lead to differences between this version and the Version of Record. Please cite this article as doi: $10.1111 /$ liv.13217

This article is protected by copyright. All rights reserved. 
Abstract: 221 words.
Abbreviations :
TKI : Tyrosine kinase inhibitor
mCRC : Metastatic colorectal cancer.
CTCAE : Common toxicity criteria for Adverse Events
ALT : Alanine aminotransferase.
AST : Aspartate aminotransferase.
LFT : Liver function tests
CYP3A4 : Cytochrome P 3A4.
UGT1A9 : Uridine diphosphate-glucuronosyltransferase 1A9.
GGT : Gamma-glutamyltranspeptidase.
ALP : Alkaline phosphatase.
LDH : Lactate dehydrogenase.
INR $\quad$ : International normalized ratio.
IHC : Immunohistochemical
HEV : Hepatitis E virus
DILI : Drug-induced liver injury.

\section{Conflicts of interest:}

None.

Financial support:

None.

This article is protected by copyright. All rights reserved. 


\begin{abstract}
BACKGROUND: Regorafenib is the first small-molecule multikinase inhibitor which showed survival benefits in pretreated metastatic colorectal cancer (mCRC) patients. Beside classical adverse events of this drug class, hepatotoxicity has been described as a frequent side effect.
\end{abstract}

MATERIAL AND METHODS: Patients with refractory mCRC treated with regorafenib in our institution were reviewed. Severe treatment-related liver toxicity was investigated. Clinical history, liver histology and genetic assessment (sequence analysis) of cytochrome $\mathrm{P}$ 3A4 (CYP3A4) and uridine diphosphate-glucuronosyltransferase 1A9 (UGT1A9) involved in regorafenib metabolization were here reported for patients with severe hepatotoxicity.

RESULTS: Among the 93 reviewed patients, 3 presented severe and icteric toxic hepatitis which was fatal for 1 patient. Histopathological liver lesions were different depending on the onset of hepatotoxicity (acute or subacute): acinar zone 3 necrosis in case of acute symptoms and portal tract inflammation with porto-central bridging and fibrosis in the delayed presentation. None patients had CYP3A4 gene mutations. Similar polymorphisms in UGT1A9 gene promoter region (UGT1A9 variant $\left.-118 \mathrm{~T}_{9>10}(\mathrm{rs} 3832043)\right)$ were found in both patients who presented acute hepatitis. Moreover, it appears retrospectively that both of them already experienced significant toxicity under irinotecan-based chemotherapy.

CONCLUSION: This is the first report of severe hepatotoxicity with available liver histology and genetic assessment of enzymes involved in regorafenib metabolization. This report also reminds the importance of a close liver tests monitoring during regorafenib treatment.

\title{
KEY WORDS:
}

Regorafenib - Metastatic colorectal cancer - Hepatotoxicity - Liver histology 


\section{KEY POINTS}

- Regorafenib showed survival benefits in pretreated metastatic colorectal and is widely used.

- Hepatotoxicy is a frequent side effect, severe liver injury is rare but grave.

- Histopathological lesions of severe liver injury show acinar zone 3 necrosis in case of acute onset and portal tract inflammation with porto-central bridging and fibrosis in the delayed presentation.

- Genetic assessment of UGT1A9 and CYP3A4 (gene involved in regorafenib metabolization) reveals similar polymorphisms in UGT1A9 gene promoter region (UGT1A9 variant $\left.-118 \mathrm{~T}_{9>10}(\mathrm{rs} 3832043)\right)$ associated with the acute form presentation.

\section{BACKGROUND}

Regorafenib is a novel orally administered tyrosine kinase inhibitor (TKI) targeting different protein kinases associated with angiogenesis, oncogenesis and maintenance of the tumor microenvironment. It showed survival benefits in pretreated metastatic colorectal cancer (mCRC) patients. In 2013, based on results of phase III trial CORRECT [1], regorafenib gained approval by the European Medicines Agency for pretreated mCRC patients.

Beside classical adverse events (fatigue, hypertension, hand-foot skin reaction and diarrhea), hepatotoxicity has been described as a side effect. In the CORRECT study, Common Toxicity Criteria (4.0) for Adverse Event (CTCAE) grade $\geq 3$ hyperbilirubinemia was observed in $2 \%$ patients, and no other adverse events associated with liver dysfunction were reported. In the CONCUR study (Asian population only), liver dysfunction was more frequent with grade $\geq 3$ hyperbilirubinemia, alanine aminotransferase (ALT) and aspartate 
aminotransferase (AST) elevation of $7 \%, 7 \%$ and $6 \%$ respectively [2]. As the mechanism of this liver toxicity remains unclear, we focused here on hepatotoxicity occurring during regorafenib treatment to extensively characterize severe case of patients with liver injury.

\section{MATERIAL AND METHODS}

Patients with refractory mCRC treated with regorafenib $(160 \mathrm{mg} /$ day 3 weeks on each 4 week cycle) in the Cliniques Universitaires Saint-Luc between January 2011 and June 2015 were reviewed. All these patients gave their consent to be treated in clinical trial [1] or in early access program before regorafenib was accessible in Belgium. Our objective was to describe the occurrence of severe treatment-related liver toxicity defined by at least an increase of bilirubin and ALT / AST to a grade 3 or 4 (CTCAE v4.0). We report here the detailed clinical history, liver function tests (LFT) and liver histology (biopsy) of identified patients. Given the hepatic metabolism of regorafenib via cytochrome P 3A4 (CYP3A4) and uridine diphosphate-glucuronosyltransferase 1A9 (UGT1A9), the most frequent mutations in both genes assessed by sequence analysis were also analyzed and reported. Approval for this research was obtained from the ethic committees of the Cliniques Universitaires Saint-Luc

\section{RESULTS}

Among the 93 patients treated with regorafenib, transient CTCAE grade 1-2 hyperbilirubinemia, ALT and AST elevation were observed during the first 2 cycles (2 months) in 21 patients $(22.5 \%), 10$ patients $(10.7 \%)$ and 11 patients $(11.8 \%)$ respectively. All patients had normal LFT (bilirubin, ALT and AST) before treatment.

This article is protected by copyright. All rights reserved. 
Three patients (3.2\%) presented severe toxic hepatitis (CTCAE grade 3-4 bilirubin, AST and ALT elevation):

- Patient 1, a 66 year-old Caucasian female, has been treated with successive treatments, including irinotecan, oxaliplatin, fluoropyrimidine, bevacizumab, panitumumab and cetuximab for mCRC with lungs, left adrenal gland and right kidney metastases. She received. Regorafenib (160 mg/day) was prescribed after disease progression. LFT were normal at the beginning of the treatment. However, 2 weeks after starting regorafenib, the patient presented with fatigue, anorexia and jaundice. Blood test revealed elevated liver enzymes: ALT 3209 U/1 (N <50), AST 2379 U/l ( $<50)$, gamma-glutamyltranspeptidase (GGT) $251 \mathrm{U} / 1(\mathrm{~N}<50)$, alkaline phosphatase (ALP) $234 \mathrm{U} / \mathrm{L}$ (N 30-120), and lactate dehydrogenase $(\mathrm{LDH})$ 841U/L $(\mathrm{N}<248)$ and total bilirubin $8.4 \mathrm{mg} / \mathrm{dl}(\mathrm{N} 0.3-1.2)$. Except regorafenib, she was not taking any other drug. Viral and autoimmune serologies were negative (Supplementary Table 1). Liver ultrasound examination was normal. Regorafenib was definitely withdrawn and LFT gradually decreased up to normal range after 5 weeks. No liver biopsy was performed. Patient died from progressive tumoral disease 10 months after fully recovered of this toxic hepatitis.

- Patient 2 was a 68 year-old Caucasian male who has been treated for KRAS mutated mCRC with peritoneal carcinomatosis. After failure of FOLFOX (folinic acid, fluorouracil, oxaliplatin) and FOLFIRI (folinic acid, fluorouracil, irinotecan) - bevacizumab, a third line therapy with regorafenib (160 mg/day) was initiated. LFT were normal before starting regorafenib. Two weeks after starting treatment, the patient developed cutaneous jaundice, dark urine, anorexia and changes in sleep patterns. LFT were dosed: ALT $3268 \mathrm{U} / 1(\mathrm{~N}<50)$, AST 3599 U/1 (N <50), GGT 187 U/l (N <50), ALP 294 U/L (N 30-120), LDH 947 U/L (N $<248$ ) and total bilirubin $19.0 \mathrm{mg} / \mathrm{dl}(\mathrm{N} 0.3-1.2)$. International normalized ratio (INR) was 4.48 (N 0.8-1.3), factor V 25\% (N 70-140), albumin 3g/dl (N3.5-5.2), blood glucose 97mg/dl 
(N 70-110). Other daily medications were simvastatin, bisoprolol, apixaban and lisinopril, all of these having been introduced more than 6 months ago. Regorafenib and all other treatments were stopped. Viral and auto-immune serological tests were negative except for a low non-specific antinuclear antibody positivity in the absence of $\operatorname{IgG}$ increase (Supplementary Table 1). Liver ultrasound did not detect the presence of hepatic metastases, nor biliary obstruction. In the context of acute hepatitis, high dose intravenous acetylcysteine was initiated. Corticotherapy was also given for 8 days. Although regorafenib was promptly withdrawn, LFT kept worsening (week 2: bilirubin $35.4 \mathrm{mg} / \mathrm{dl}$, INR 3.3), leading to progressive worsening of encephalopathy and fatal liver failure 5 weeks after treatment initiation. A postmortem percutaneous liver biopsy was performed. Histology showed centrilobular hepatic necrosis with confluent destruction of acinar zones 3. Portal tracts areas were preserved and inflammation was scarce (Fig. 1A). Immunohistochemical (IHC) and molecular tests were negative for hepatitis E virus (HEV) (Supplementary Table 2)

- Patient 3, a 68-year old Caucasian female, was suffering from KRAS mutated mCRC with lung and liver metastases. She had been successively treated with capecitabineoxaliplatine - bevacizumab and FOLFIRI. Regorafenib was initiated at full dose. LFT were normal at the beginning of treatment. Due to significant asthenia and anorexia, the dosage was reduced to $120 \mathrm{mg}$ per day from the second cycle. After 2 months, a radiologic evaluation showed a stable disease. After 4 months of treatment, elevated LFT were found on routine blood test: ALT $1738 \mathrm{U} / \mathrm{l}(\mathrm{N}<50)$, AST $1347 \mathrm{U} / 1(\mathrm{~N}<50)$, GGT $624 \mathrm{U} / 1(\mathrm{~N}<50)$, ALP 296 U/L (N 30-120), LDH 482U/L ( $<248)$ and total bilirubin 5.2 mg/dl (N 0.3-1.2). Autoimmune and viral serologies were negative (Supplementary Table 1). No others hepatotoxic drugs were taken. Regorafenib was withdrawn and high dose intravenous acetylcysteine was administered. A transjugular liver biopsy was performed. The hepatovenous gradient was $4 \mathrm{mmHg}$. Liver histology showed severe portal tract inflammation 
with porto-central bridging, fibrosis and hepatocytes loss in the central area (Fig. $1 B)$. IHC and molecular testings were negative for HEV (Supplementary Table 2). LFT progressively improved with normalisation within 2 months after stopping the treatment. Patient died from progressive tumoral disease 6 months after fully recovered of this complication.

Given the hepatic metabolism of regorafenib via CYP3A4 and UGT1A9, the most frequent mutations in both 2 genes were assessed by sequence analysis. Patient 1 and patient 2 carried similar genetic polymorphisms in the UGT1A9 gene promoter region. Both were heterozygous for UGT1A9*22 allele (UGT1A29 variant $-118 \mathrm{~T}_{9>10} ; \quad$ rs3832043), characterized by a single base insertion of thymidine. Moreover, a heterogenous and homogenous intronic transversion $(\mathrm{C}>\mathrm{T})$ were identified in patients 1 and 2 respectively. UGT1A9 sequencing did not show any mutation in patient 3. Regarding the CYP3A4 gene, no mutation was discovered in any of the patients.

Patients' characteristics are summarized in Table $\mathbf{1 .}$

\section{DISCUSSION}

The introduction of small-molecule TKIs in clinical oncology has transformed the treatment of certain forms of cancers. Unexpectedly, their use has been found to be associated with serious toxic effects on a number of vital organs including the liver. Indeed, hepatotoxicity in cancer patients treated with TKI is well reported and justifies close monitoring: a meta-analysis demonstrated a significant increase of developing high-grade hepatotoxicity with TKIs use [3-4].

This article is protected by copyright. All rights reserved. 
The regorafenib safety profiles were initially limited before investigators decided to proceed to a phase III trial due to positive signals observed in phase I [5]. Although less predictable, elevated LFT were also frequent and generally occurred soon after treatment initiation. Therefore, monitoring of bilirubin and transaminases was considered as crucial during the first two cycles in order to allow early discontinuation in case of liver abnormalities. Severe drug-induced liver injury (DILI) led to fatal outcome in only $0.3 \%$ of 1100 regorafenib-treated patients across all clinical trials [6] and all patients presenting with hepatic failure also had liver metastases.

In our trial, the proportion of patients with grade 1-2 increased bilirubin or aminotransferases was higher as reported in the CORRECT trial [1]. Here, we reported severe hepatoxicity for 3 patients including one patient with fatal outcome. We distinguish two clinical histories of hepatitis, each of them associated with different histopathological patterns. In patient 3, biochemical abnormalities and symptoms appeared several months after initiation of the treatment, and liver biopsy showed features of severe active hepatitis with portal inflammation, bridging fibrosis, reminding of auto-immune hepatitis. By contrast, in patient 2, we found centrilobular necrosis, a morphological abnormality seen in acute acetaminophen toxicity, whose mechanism is also linked to the P-450 cytochrome system.

Regorafenib is a major substrate of CYP3A4, a factor described as an important predictor of DILI [7]. Furthermore, given its exclusive liver metabolism, regorafenib is a medication at higher risk for DILI [8]. In the patient 2 case, regorafenib was coadministred with three other CYP3A4 substrates (simvastatin, bisoprolol and apixaban) but all of them were already introduced since several months. Considering that more than one substrate can 
bind to the active enzyme site of CYP3A4 [9] and the clear evidence for cooperativity in substrate binding and in catalytic turnover [10], the influence of other CYP3A4 substrates on regorafenib metabolism could have been a factor contributing to the severe toxicity observed in patient 2 .

UGT1A9 is the second enzyme implicated in regorafenib metabolism. Over the last decade, polymorphism in UGT1A superfamily has been widely studied, particularly in order to predict interindividual toxicity of irinotecan [11]. Moreover, a recent Chinese study showed an association between UGT1A9 promoter polymorphisms ( $\mathrm{T}>\mathrm{C}$ transition; position-440 (rs2741045)) and DILI [12] but no pieces of evidence demonstrated so far the functional impact of $-118 \mathrm{~T}_{9>10}$ polymorphism on interindividual variation in hepatic UGT1A9 activity and mRNA expression [13]. In the present report, it is of note that both patients presenting with acute hepatic dysfunction exhibited similar polymorphisms (variant $\left.118 \mathrm{~T}_{9>10}\right)$ in UGT1A9 gene promoter region. Retrospectively, it appears that both of them experienced intolerance under irinotecan-based chemotherapy requiring fast dose reduction: grade 3 neutropenia during the first cycle for patient 1 and grade 3 neutropenia and diarrhea for patient 2 .

Our report remains descriptive with several limitations that cannot confirm the definitive role of UGT1A9 polymorphisms in regorafenib hepatotoxicity (and a possible cross-toxicity with irinotecan). Indeed, UGT1A9 genotyping was only performed in the 3 patients with severe liver injury and not in the remaining cohort. Moreover, the reported high frequency (up to $39 \%$ of patients) and lack of interindividual liver activity variation of the $-118 \mathrm{~T}_{9>10}$ polymorphism justifies caution and further reports before any conclusion.

Another limitation is the absence of histology available for patient 1. However, acute presentation following regorafenib introduction and return to normal after treatment cessation 
are in favor of DILI. HEV is a relevant differential diagnosis to DILI. Since patient's residual sera were not available, we applied combined IHC and molecular testing for HEV on liver biopsies and found no evidence for a HEV infection (Supplementary Table 2).

To our knowledge, this is the first report of severe hepatotoxicity with available liver histology and genetic assessment of enzymes involved in regorafenib metabolization. Conversely to the published case-reports [14-16], one of our patients died from liver failure. LFT from others patients improved with normalisation as described in these reports. Furthermore, our patients had no previous history of liver dysfunction. Both of these arguments advocate a direct toxicity of the multikinase inhibitor without predisposing liver condition.

In summary, this report highlights the importance of a close liver monitoring during regorafenib treatment, especially during the first cycles. Nevertheless, even if infrequent, delayed hepatic toxicity can occur. It illustrates also that severe hepatotoxicity is not limited to patients presenting with liver metastases. Whereas the mechanism of toxicity remains so far unclear, further reports are needed to explore the role of CYP3A4 and UGT1A9 polymorphisms and hepatic activities and the possible cross-toxicity with irinotecan...

\section{REFERENCES}

[1] Grothey A, Van Cutsem E, Sobrero A, Siena S, Falcone A, Ychou M, et al. Regorafenib monotherapy for previously treated metastatic colorectal cancer (CORRECT): an international, multicentre, randomised, placebo-controlled, phase 3 trial. Lancet 2012;381(9863):303-312.

[2] Li J, Qin S, Xu R, Yau TC, Ma B, Pan H et al. Regorafenib plus best supportive care versus placebo plus best supportive care in Asian patients with previously treated metastatic 
colorectal cancer (CONCUR): a randomised, double-blind, placebo-controlled, phase 3 trial. Lancet Oncol 2015;16(6):619-29.

[3] Lacovelli R, Palazzo A, Procopio G, Santoni M, Trenta P, De Benedetto A et al. Incidence and relative risk of hepatic toxicity in patients treated with antiangiogenic tyrosine kinase inhibitors for malignancy. $\mathrm{Br} J$ Clin Pharmacol. 2014;77(6):929-38.

[4] Teo YL, Ho HK, Chan A. Risk of tyrosine kinase inhibitors-induced hepatotoxicity in cancer patients: a meta-analysis. Cancer Treat Rev. 2013;39(2):199-206.

[5] Strumberg D, Scheulen ME, Schultheis B, Richly H, Frost A, Büchert M, et al. Regorafenib (BAY73-4506) in advanced colorectal cancer: a phase I study. Br J Cancer. 2012;106(11):1722-1777.

[6] http://www.accessdata.fda.gov/drugsatfda_docs/label/2012/203085lbl.pdf - Highlights of prescribing information about Stivarga.

[7] Yu K, Geng X, Chen M, Zhang J, Wang B, Ilic K, et al. High daily dose and being a substrate of cytochrome P450 enzymes are two important predictors of drug-induced liver injury. Drug Metab Dispos 2014;42:744-750.

[8] Lammert C, Bjornsson E, Niklasson A, Chalasani N. Oral medications with significant hepatic metabolism at higher risk for hepatic adverse events. Hepatology 2010;51:615-620.

[9] Atkins WM. Non-Michaelis-Menten kinetics in cytochrome P450-catalysed reactions. Annu Rev Pharmacol Toxicol 2005;45:291-310.

This article is protected by copyright. All rights reserved. 
[10] Fernando H, Halpert JR, Davydov DR. Resolution of multiple substrate binding sites in cytochrome P450 3A4: the stoichiometry of the enzyme-substrate complexes probed by FRET and Job's titration. Biochemistry 2006;45:4199-4209.

[11] Cecchin E, Innocenti F, D’Andrea M, Corona G, De Mattia E, Biason P, et al. Predictive role of the UGT1A1, UGT1A7 and UGT1A9 genetic variants and their haplotypes on the outcome of metastatic colorectal cancer patients treated with fluorouracil, leucovorin and irinotecan. J Clin Oncol 2009;27:2457-2465.

[12] Jiang J, Zhang X, Huo R, Li X, Yang Y, Gai Z, et al. Association study of UGT1A9 promoter polymorphisms with DILI based on systematically regional variation screen in Chinese population. Pharmacogenomics J 2015;15:326-331.

[13] Ramirez J, Liu W, Mirkov S, Desai AA, Chen P, Das S, et al. Lack of Association between Common Polymorphisms in UGT1A9 and Gene Expression and Activity. Drug Metab Dispos. 2007;35:2149-53.

[14] Akamine T, Ando K, Oki E, Saeki H, Nakashima Y, Imamura YU,et al. Acute liver failure due to regorafenib may be caused by impaired liver blood flow: a case report. Anticancer Res. 2015;35: 4037-41.

[15] Raissouni S, Quraishi Z, Al-Ghamdi M, Monzon J, Tang P, Vickers MM. Acute liver failure and seizures as a consequence of regorafenib exposure in advanced rectal cancer. BMC Res Notes. 2015;8:538.

[16] Kuwayama M, Uchino K, Takayoshi K Komoda M, Kohjima M, Nakamuta M, et al. Immunosuppressant therapy successfully improved regorafenib-induced severe hepatic injury in a patient with metastatic gastrointestinal stromal tumor: A case report. Oncol Lett 2016;11(1):85-88

This article is protected by copyright. All rights reserved. 


\section{Figure legends}

Fig. 1A: Post mortem liver biopsy from patient 2, hematoxylin-eosin, original magnification X8. There is extensive necrosis around the central vein $(*)$ with confluent destruction of acinar zones 3 (arrow heads). By contrast, the portal tracts (PT) areas are mostly preserved.

Fig. 1B: Transjugular liver biopsy from patient 3, Masson's Trichrome, original magnification X12. Severe portal tract inflammation together with interface hepatitis and porto-central bridging is observed (arrow). Centrolobular areas (upper left) show inflammation and hepatocytes loss.

Table 1: Patient's characteritics

\section{Patient 1}

66-year old female

Absence

No

Presence of liver metastases

Toxicity under FOLFIRI

Time between start regorafenib and hepatotoxicity

Grade III neutropenia during first cycle
Patient 2

Patient 3

68-year old male

68-year old female

Presence

Presence

No

Yes

Grade III

No

neutropenia and diarrhoea

2 weeks

4 months

This article is protected by copyright. All rights reserved. 
Liver histology

Evolution after discontinuing regorafenib

UGT1A9 gene mutations mutations
Normalisation within 5 weeks

Heterozygous -118 (dT)10:

UGT1A1*1B

Heterozygous -331

$\mathrm{C}>\mathrm{T}$

No
Centrilobular hepatic necrosis with confluent destruction of acinar zones 3
Severe portal tract inflammation with porto central fibrosis

Liver failure leading Normalisation within to death after $3 \quad 5$ weeks weeks

Heterozygous-118
(dT)10:
UGT1A1*1B
Homozygous -331
C $>$ T

No
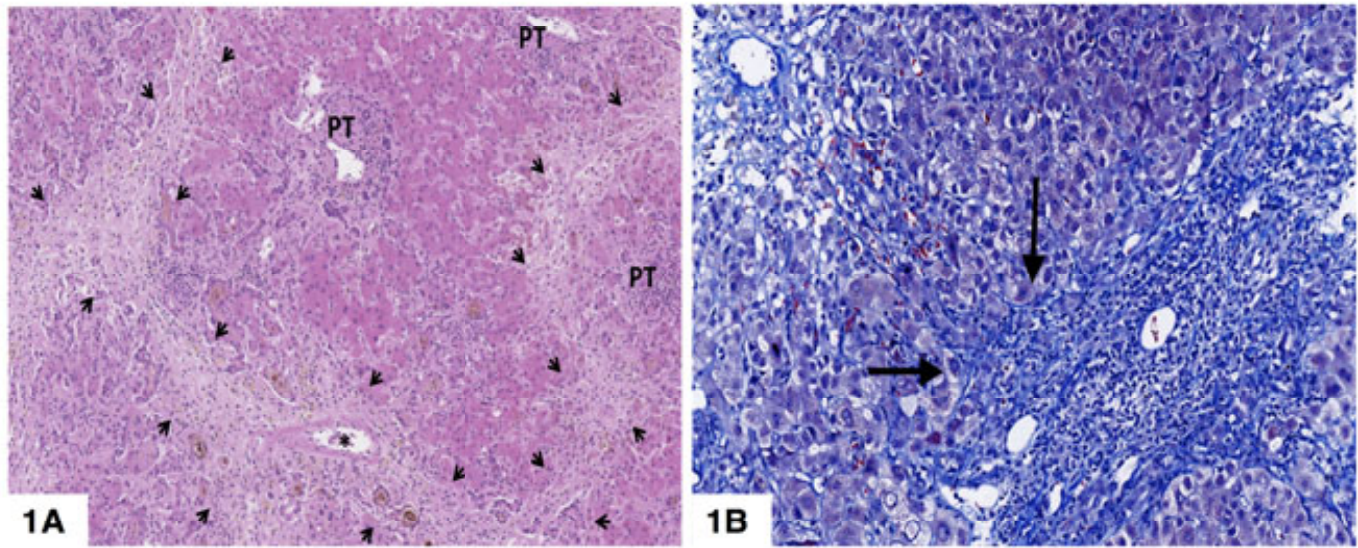

This article is protected by copyright. All rights reserved. 